\title{
Numerical Simulation of the Blood Flow through a Brain Vascular Aneurysm with an Artificial Stent Using the SPH Method
}

\section{Leonardo Di G. Sigalotti' ${ }^{1}$, Jaime Klapp², Karla Pedroza ${ }^{3}$, Edgar Nathal4, Carlos E. Alvarado-Rodríguez ${ }^{5}$}

${ }^{1}$ Area of Physics of Irreversible Processes, Department of Basic Sciences, Autonomous Metropolitan University-Azcapotzalco (UAM-A), Mexico City, Mexico ${ }^{2}$ Physics Department, National Institute of Nuclear Research (ININ), Ocoyoacac, Mexico

${ }^{3}$ Los Cabos H+ Hospital, San José del Cabo, Mexico

${ }^{4}$ National Institute of Neurology and Neurosurgery "Manuel Velasco Suárez", Mexico City, Mexico

${ }^{5}$ Departament of Chemical Engineering, DCNyE, University of Guanajuato, Guanajuato, Mexico

Email: leonardo.sigalotti@gmail.com,jaime.klapp@inin.gob.mx, iqcarlosug@gmail.com

How to cite this paper: Sigalotti, L.D.G., Klapp, J., Pedroza, K., Nathal, E. and Alvarado-Rodríguez, C.E. (2018) Numerical Simulation of the Blood Flow through a Brain Vascular Aneurysm with an Artificial Stent Using the SPH Method. Engineering, 10, 891-912.

https://doi.org/10.4236/eng.2018.1012062

Received: November 19, 2018

Accepted: December 25, 2018

Published: December 28, 2018

Copyright $\odot 2018$ by authors and Scientific Research Publishing Inc. This work is licensed under the Creative Commons Attribution International License (CC BY 4.0).

http://creativecommons.org/licenses/by/4.0/

\begin{abstract}
We present numerical simulations of blood flow through a brain vascular aneurysm with an artificial stent using Smoothed Particle Hydrodynamics $(\mathrm{SPH})$. The aim of this work is to analyze how the flow into an aneurysm changes using different stent configurations. The initial conditions for the simulations were constructed from angiographic images of a real patient with an aneurysm. The wall shear stresses, pressure and highest velocity within the artery, and other particular quantities are calculated which are of medical specific interest. The numerical simulations of the cerebral circulation help doctors to determine if the patient's own vascular anatomy has the conditions to allow arterial stenting by endovascular method before the surgery or even evaluate the effect of different stent structure and materials. The results show that the flow downstream the aneurysm is highly modified by the stent configuration and that the best choice for reducing the flow in the aneurysm is to use a completely extended Endeavor stent.
\end{abstract}

\section{Keywords}

Brain Vascular Flow, Aneurysms, Blood Flow, Particle Methods, Numerical Modeling 


\section{Introduction}

Brain vascular pathologies are difficult to treat and have a broad field of research. A brain aneurysm is a vessel dilation caused by a weakness of the artery's wall, with risk of rupture or cerebral compression when reaching a big size. But even with the best technology many patients have lost the opportunity to cure because of a special manifestation of the disease. In these cases, simulation of the angioarchitecture is crucial for planning the treatment. The general incidence of bleeding related to an aneurysm rupture is $9.1 / 100,000$ persons/year, but there are countries with two times more risk of bleeding like Japan with 22.7/100,000 persons/year [1].

There are different shapes of aneurysms, but in general they can be classified as saccular and fusiform. The first one is the most common form in $90 \%$ of cases. Several studies have found that the localization, size and shape are important factors of rupture, a trial called ISUIA (International Study of Unruptured Intracranial Aneurysms). A critical review of the ISUIA found that aneurysms greater than $10 \mathrm{~mm}$ and located at posterior circulation have more risk of rupture [2] as well as a long perpendicular height (the measurement of the aneurysm height at a perpendicular direction from the center of the aneurysm neck to the aneurysm dome) [3] [4]. The standard treatment of brain aneurysm ruptured or unruptured are open surgery with clipping or revascularization and endovascular therapy that can be with coiling or stenting [5] [6] [7]. Most of the aneurysms that can be treated by endovascular methods are candidates to embolization with coils which are small pieces of platine that fill the aneurysm cavity avoiding the blood stress on the aneurysm wall. However, not all the aneurysms can be treated in that way. For instance, this is the case of fusiform aneurysms, of saccular aneurysms with wide neck and giant aneurysms that are very big to be filled by coils, which must be treated with surgery or with a special device called flow diverter, stents and low profile endovascular devices [8] [9] [10]. This kind of devices are like expandable cylinders put by endovascular access that cover the inner membrane of the vessels avoiding or lowering the flow of blood within the aneurysm that could eventually lead to thrombosis. The stents are built with nitinol, platinum-tungsten and cobalt, and chromium with diameters of 2.5 to $6 \mathrm{~mm}$ and lengths between 14 and $40 \mathrm{~mm}$. Depending upon the reduction of the vessel diameter, the flow and the porosity-dependent pressure, they can be classified as laser-cut or braided devices. The stents are composed of a mesh that is organized following an architecture of open-cells or closed cell [11] (see Figure 1).

There are actually a lot of questions about the best materials to employ and the design of the endovascular devices for complex aneurysm treatment. Further developments should focus on improving the deployment and the best stent for every patient. On the other hand, the brain vascular computer simulations must reproduce the vascular tree or at least some of its parts in order to estimate the response to the treatment prior to the accomplishment of a modification of the 


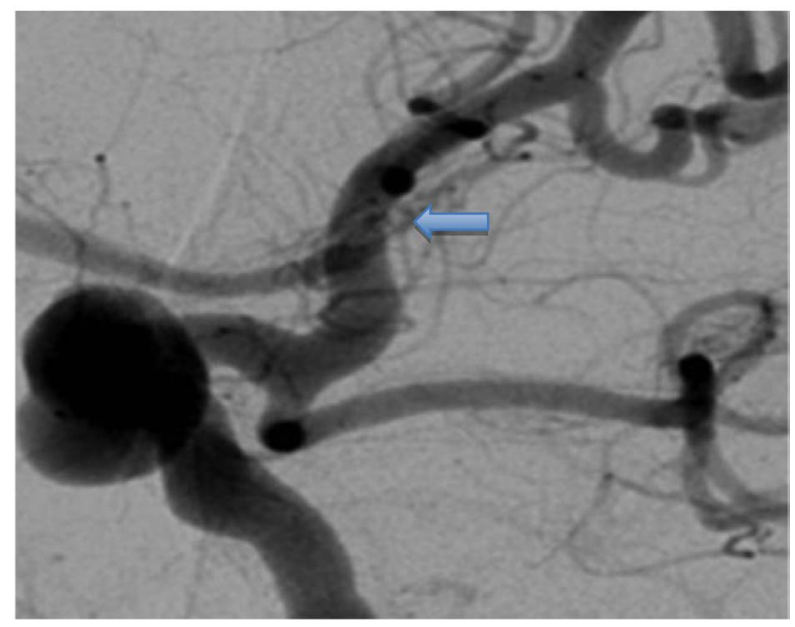

Figure 1. Stent image as seen in an angiogram. The blue arrow points to the position of the stent.

cerebral vascular anatomy. Virtual stenting may simulate the cerebral blood flow change in a vessel with aneurysmal pathology in a specific patient using the hemodynamic modification analysis with a specific device [12] as well as finite element methods [13]. In order to enhance prediction, the effects of blood viscosity, soft materials, thickness and the architecture of the complex network even in not completely expanded stents need be simulated. In this paper, the numerical simulations were performed using the method of Smoothed Particle Hydrodynamics (SPH). SPH is a particle-based, Lagrangian method used for simulating the mechanics of continuous media [14]. The basic idea behind SPH is very simple. It starts by reconstructing a continuous field at any point in space and time from a cloud of discrete particles, which are the material property carriers. This is accomplished by a three-dimensional interpolation of the scattered data by means of a kernel function [14]. Medical applications using the SPH method have started to appear very recently. For example, interactive blood simulations for virtual surgery using the SPH method have been performed by Muller et al. [15], while SPH of left ventricular blood flow has been reported by Caballero et al. [16]. Although, computational hemodynamic analyses of endovascular devices have been performed using conventional methods [17] [18] [19], so far no simulations with SPH exist in the literature. The initial conditions for the SPH simulations are constructed using angiographic images of a real patient with an aneurysm. While this is the gold standard test for obtaining the best quality in angioarchitecture, such images can also be obtained from a CT scan or a MRI with 3D reconstruction [20] [21], as shown in Figure 2.

The paper is organized as follows. A description of the numerical models and parameters are given in Section 2. Section 3 describes the main results, and Section 4 contains the relevant conclusions.

\section{Numerical Models and Parameters}

The numerical simulation of blood flow through a brain vascular aneurysm with 


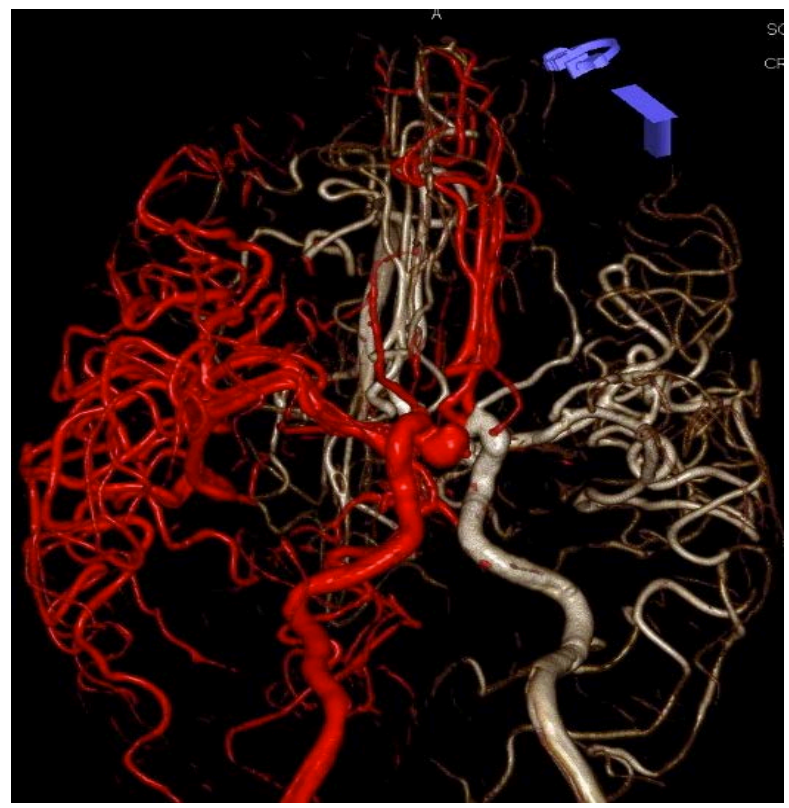

Figure 2. Patient angiography with 3D reconstruction.

an artificial stent was performed using the SPH method [14] [22]. The code solves the conservation laws of mass and momentum for a viscous fluid with prescribed boundary conditions. The discretization of the governing equations is performed over a cloud of scattered particles, which are the carriers of the fluid properties. For more details on the method, the reader is referred to Monaghan [14] and Liu and Liu [22]. The aim of this work is to analyze the hemodynamic flow as it goes into the aneurysm using different stent configurations from which it is possible to calculate the wall shear stress tensor, the pressure and the highest velocity within the artery as well as other particular quantities which may have a specific interest for medical evaluation.

The numerical simulations were performed using a modified version of the DualSPHysics code, which is described in detail by Crespo et al. [23]. One of the main advantages of the DualSPHysics code is its parallel structure. The code is written in the $\mathrm{C}++$ language using the Open Multi-processing application that allows performing calculations in parallel using different numbers of cores according to the computer equipment used. In addition, there is a version written in CUDA (Compute Unified Device Architecture) that allows the use of GPU processors (Graphics Processor Unit).

\subsection{Artery, Aneurysm and Stent Geometry}

The geometry of the aneurysm was taken from the angiographic image of a real patient as illustrated in the Figure 3 from three different perspectives, front (YZ plane), side (XZ plane) and top (XY plane). A virtual file with this geometry was produced from the results of the angiography and exported to the SPH code to create the boundary conditions of the artery and aneurysm. Figure 4 shows the distribution of boundary SPH particles (right) employed to reconstruct the true 


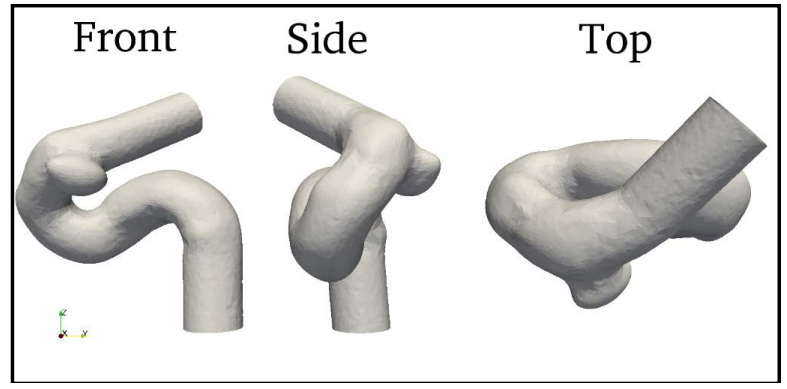

Figure 3. The aneurysm of a patient taken from an angiographic image. The image is shown from three different perspectives: front ( $\mathrm{YZ}$ plane), side (XZ plane) and top (XY plane).

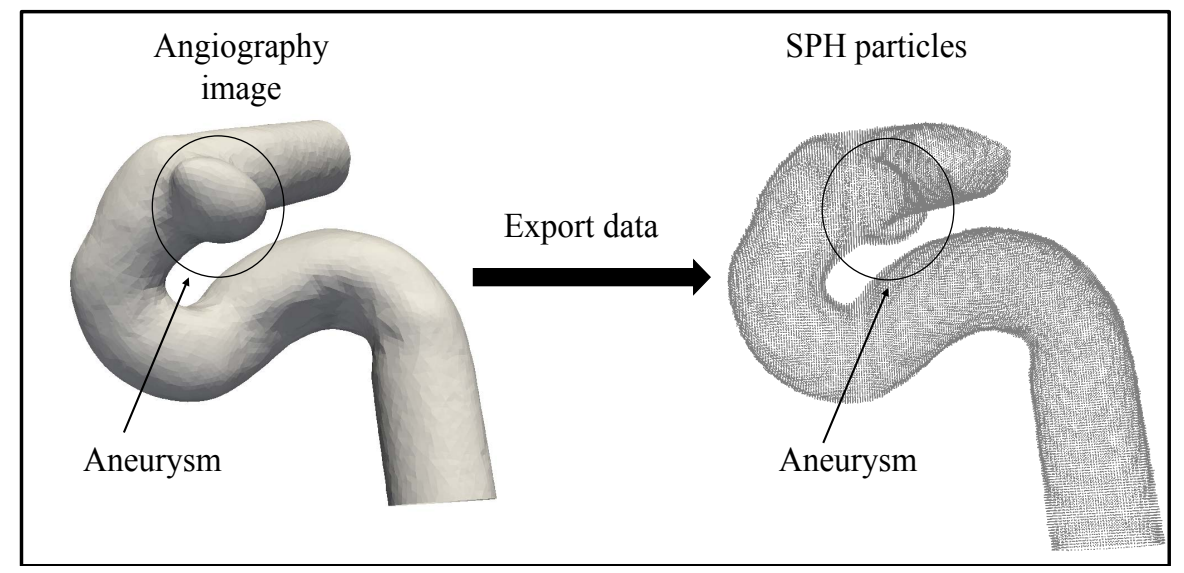

Figure 4. SPH particle reconstruction of the angiographic image of an aneurysm. The boundary of the artery is made up of SPH particles.

artery and aneurysm geometries (left). In the present simulations, the assumption is made that both the artery and the aneurysm are rigid bodies.

Two generic stent geometries were considered to analyze the flow behavior into the aneurysm when one stent is introduced into the artery. The first stent used in this study is equivalent to a second generation Endeavor stent for which the strut wall thickness is $0.09 \mathrm{~mm}$. The geometry of the stent is shown in the left frame of Figure 5. The second stent was constructed by taking an octagonal geometry as the basis of the mesh. The geometry of the octagonal stent is depicted in the right frame of Figure 5. The stent model geometries were reproduced with the aid of the software Blender v2.67 and the stent insertion into the aneurysm was made using tools of the software Blender, which creates a file that is finally imported into the DualSPHysics code to reproduce numerically the geometry of both the stent and the artery using SPH particles.

The geometry from the angiographic image was used as the basis to model both stents. Four different cases of study are considered. In the first case, the Endeavor stent is extended completely into the artery, so that it almost fits the wall boundaries of the artery as shown in the left frame of Figure 6. The second case deals with the Endeavor stent not being completely extended and so it is 

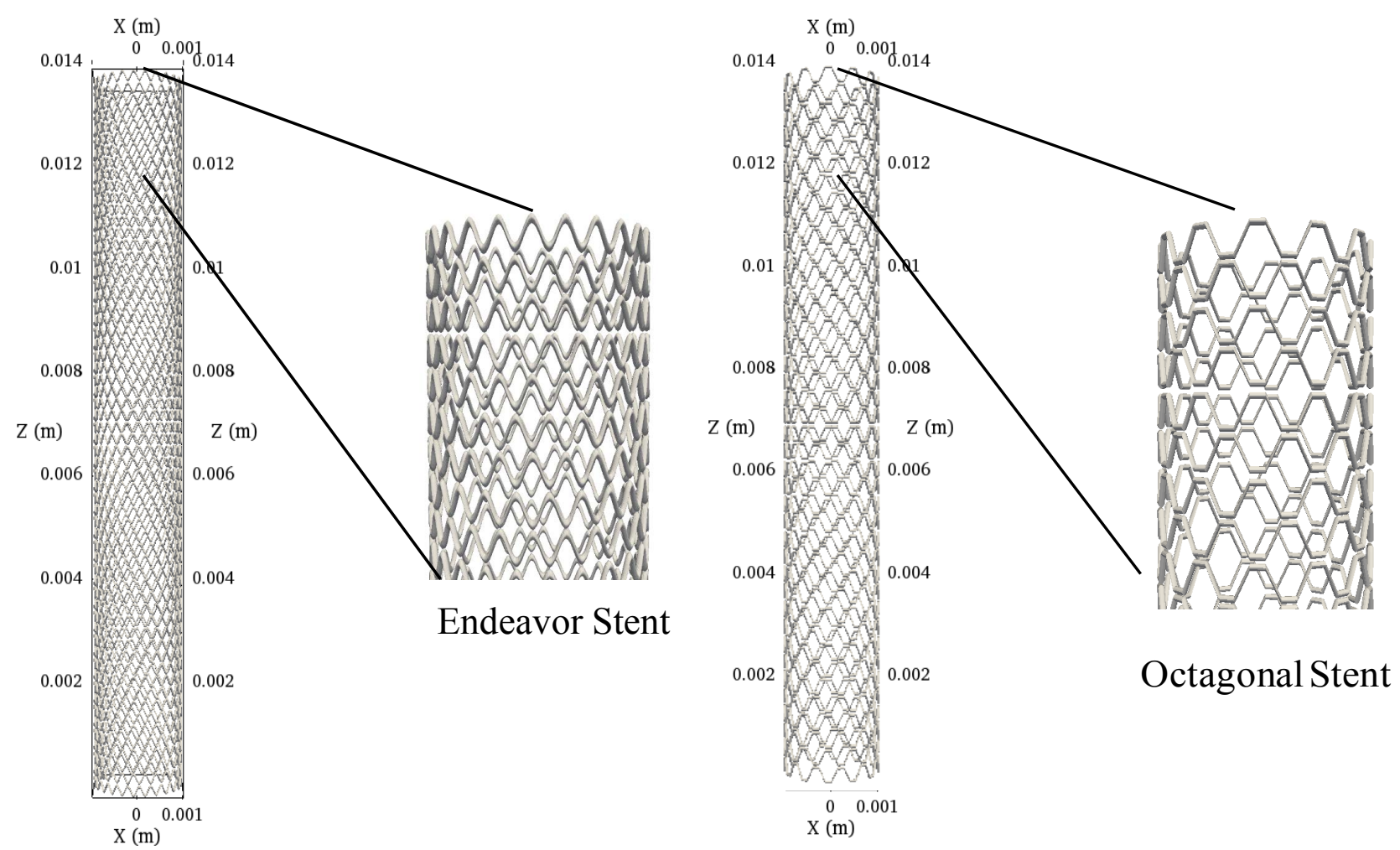

Figure 5. Geometry and dimensions of the Endeavor (left) and octagonal (right) stents as used in the numerical simulations.

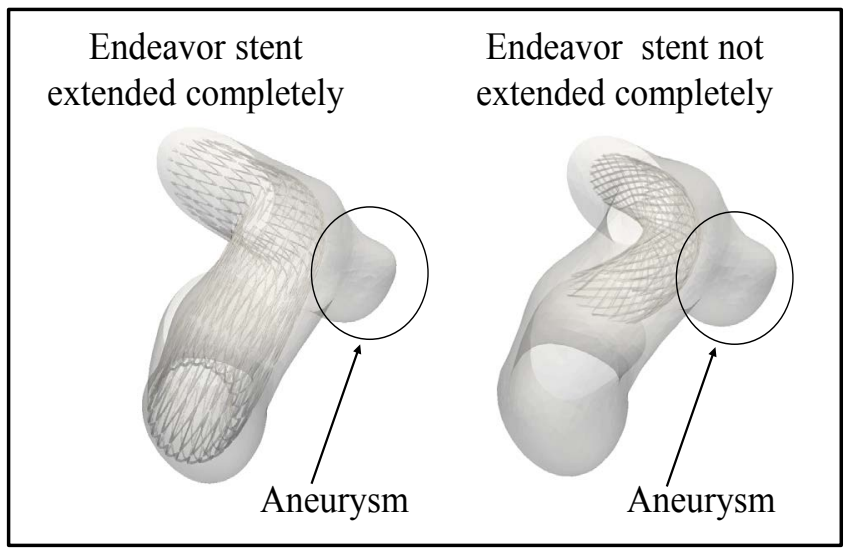

Figure 6. Completely (left) and partially extended (right) Endeavor stents within the artery as employed in the numerical simulations.

slightly separated from the wall of the artery as shown in the right frame of Figure 6.

The third and fourth cases of study maps the geometry obtained from the angiographic image with an octagonal stent into the artery. In the third case, the octagonal stent is again extended completely so that the mesh fits the inner wall of the artery, while in the fourth case the stent is not extended completely and so it looks separated from the wall of the artery. These geometries are both displayed in Figure 7. 


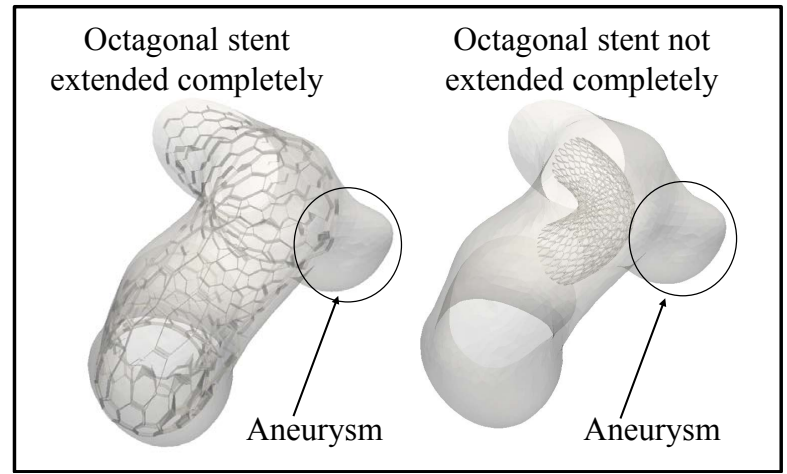

Figure 7. Completely (left) and partially extended (right) octagonal stents into the artery as employed in the numerical simulations.

\subsection{Parameters}

The parameters used in the numerical simulations are listed in Table 1.

The reference density corresponds to the average value of whole blood for a human, while $v=3.36 \mathrm{mPa}, \mathrm{s}$ is the blood dynamical viscosity at $27^{\circ} \mathrm{C}$. The background pressure of $7500 \mathrm{~Pa}$ corresponds to the normal diastolic arterial pressure. The smoothing length is the width of the interpolating kernel function used in SPH and determines the spatial resolution of the calculation. For about half million particles a value of $1.732 \mathrm{~mm}$ is enough for resolving small features of the flow and representing the arterial irregularities with sufficiently good accuracy. The time duration of a simulation is typically 6.0 seconds. At the initial time ( $t=0 \mathrm{~s}$ ), the blood within the aneurysm is assumed to be at rest (i.e., $v=0$ ).

\subsection{Boundary Conditions}

No slip boundary conditions are used at the solid wall of the artery, using the dynamic particle method implemented in the DualSPHysics code [23]. At the inlet of the aneurysm the flow velocity is set by a sequential flow rate characterizing a systole and diastole pulsatile flow wave in the cardiac cycle, the two mean phases of each heartbeat. The flow rate at the inlet corresponding to this pulsatile blood flow is shown in the right frame of Figure 8. Non-reflecting outlet boundary conditions, as were recently developed for pipe flow simulations by Alvarado-Rodríguez et al. [24], were implemented at the outlet of the artery. This outflow boundary conditions are implemented using an outflow zone downstream of the outlet, where particles are moved using an outgoing wave equation for the velocity field so that feedback noise from the outlet boundary is greatly reduced. The inlet and outlet flow geometry is depicted in the left frame of Figure 8.

\section{Results}

The results of the numerical simulations are divided into three parts. In the first part, we describe the results in terms of the blood flow rate at the inlet and outlet 
Table 1. Parameters used for the numerical simulations.

\begin{tabular}{cc}
\hline Parameter & Value \\
\hline Reference density $\left(\rho_{0}\right)$ & $1056 \mathrm{~kg} \cdot \mathrm{m}^{-3}$ \\
Dynamical viscosity $(v)$ & $3.36 \mathrm{mPa} \cdot \mathrm{s}$ \\
Speed of sound $(c)$ & $40 \mathrm{~m} \cdot \mathrm{s}^{-1}$ \\
Background pressure $(p)$ & $7500 \mathrm{~Pa}$ \\
Smoothing length $(h)$ & $1.732 \times 10^{-3} \mathrm{~m}$ \\
Gravity $(g)$ & $-9.8 \mathrm{~m} \cdot \mathrm{s}^{-2}$ \\
CFL number & 0.2 \\
Total number of particles & 493,904 \\
\hline
\end{tabular}
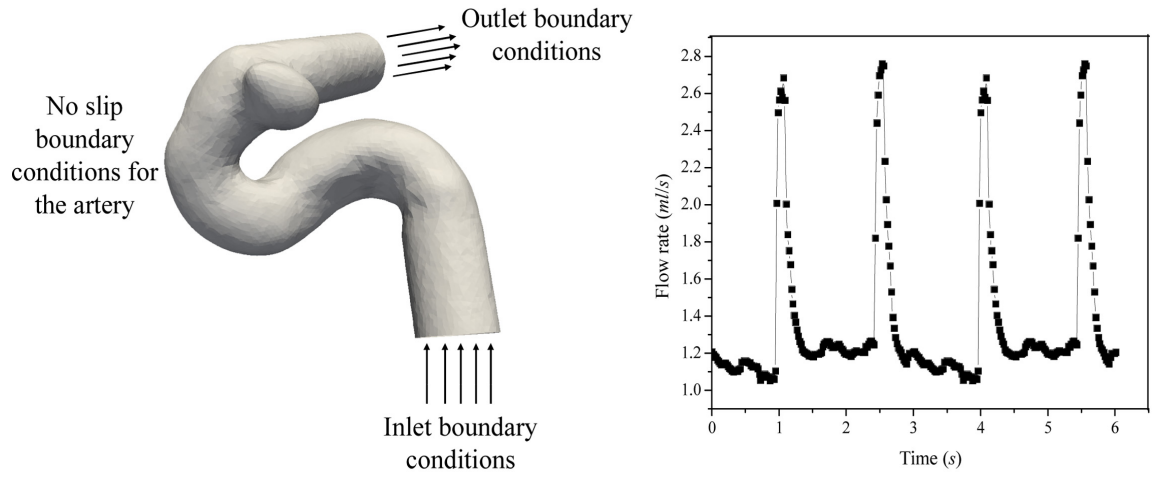

Figure 8. Boundary conditions. The inlet flow rate shown in the right frame was used as the inlet boundary conditions, while at the outlet of the artery fully non-reflecting boundary conditions are employed.

planes, the velocity at the center of the aneurysm and the velocity field through the artery for each case of study. In the second and third parts, results are given for the pressure and the wall shear stress tensor, respectively. All the numerical results were obtained using the tools of DualSPHysics and the free software ParaView 5.1.2 for large data visualization.

\subsection{Velocity and Flow Rates}

The inlet flow rate profile is the same for all cases of study. However, the flow rate profile at the center of the artery before the stent zone is modified due to the presence of the stent. The flow rates are very similar to the inlet profile for all cases except for the case with the octagonal stent when it is extended completely. This is due to the geometry of the stent and its thickness. The thickness of the stent canalizes the flow in the center of the artery by a reduction of the transversal area, thereby increasing the flow velocity. The flow rates for each case of study are seen to differ at the zone just before the stent as shown in Figure 9. The flow rate was measured at an inner station of the artery as shown by the black point of coordinates $(0,0,0.03)$.

At the outlet of the artery, the flow is also seen to be greatly affected by the 

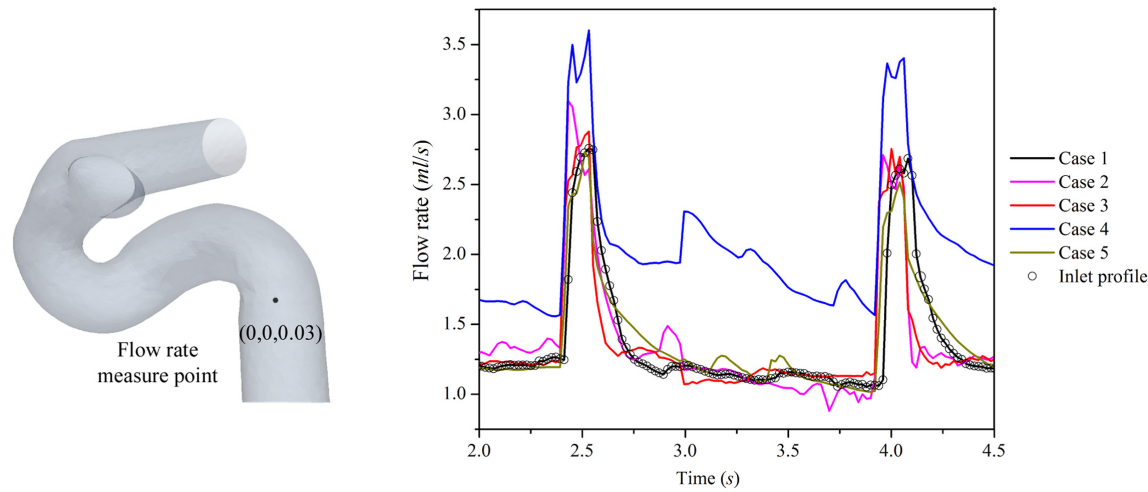

Figure 9. Flow rate profile within the artery before the stent zone (right), at the coordinate point shown in the left frame. The right frame shows how the flow rate is modified due to the stent in the artery.

stent configuration. The flow rate at the outlet of the artery should have similar characteristics to the inlet flow rate due to the artery being assumed to be a rigid body. However, the flow rate profile at the outlet, as measured at the center of the artery at the coordinate point $(-0.03,-0.03,0.125)$, is also modified by the stent configuration as shown in the right frame of Figure 10. In the simulation of the artery without stent (Case 1), the flow rate is slightly reduced by the presence of the aneurysm and the change of the transversal area of the artery, and in spite of this the flow looks very similar to that at the inlet. In all cases with stent, the flow rate at the outlet of the artery is seen to increase due to the reduction of the transversal area, while the reduction of the highest flow rates is also smoother in each cardiac cycle as shown in Figure 10.

One purpose of introducing the stent into the artery is to reduce the flow and pressure in the aneurysm. Therefore, it is important to compare the fluid velocity in the aneurysm for the cases when the stent is present to the case when the artery is free of stent. The numerical results indicate that the velocity into the aneurysm decreases by about $88.5 \%$ in the cases 2,4 and 5 compared to case 1 with no stent. The fluid velocity into the aneurysm increases just after the first pulsatile wave. However, it is not seen to decrease in a similar way as occurs close to the inlet as can be observed by comparing Figure 9 and Figure 10. These results suggest that there exists a constant flow into the aneurysm caused by turbulence (see Figure 17) that keeps a constant move in the fluid when it is flowing in the artery. On the other hand, when a stent is introduced in the artery, strong changes in the velocity are seen to occur within the aneurysm. The pulsatile flow in the aneurysm appears in those cases where the stent is not completely extended and disappears when the stent is completely extended, implying a decrease of the velocity field by about $92.8 \%$ for case 2 . In case 3 , the velocity is reduced by about $71.4 \%$ due to the design of the stent and the amplitude of the octagonal mesh. In all cases, the fluid velocity and therefore the flow is reduced in the aneurysm when a stent is introduced into the artery. However, the features of the flow are different when the stent is completely extended or not. 

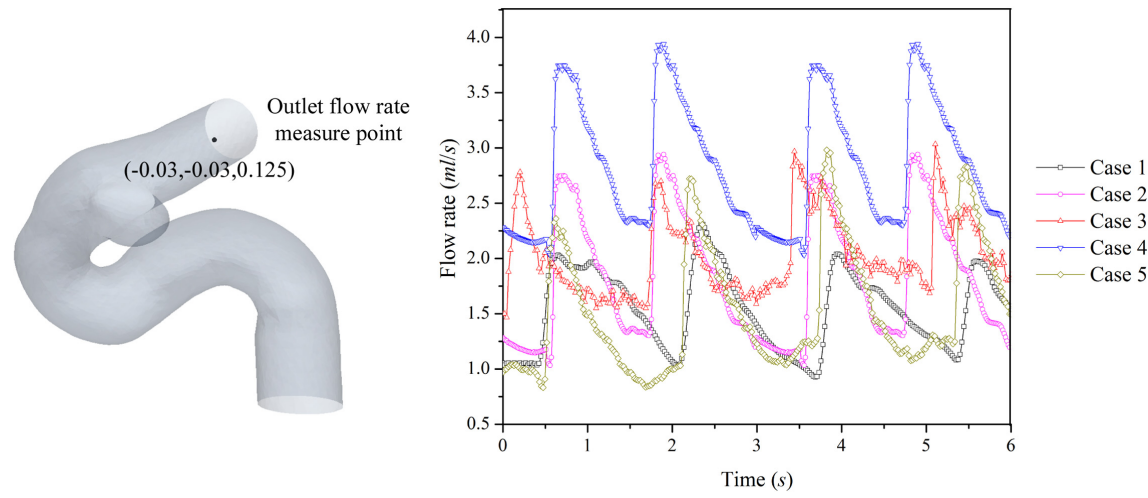

Figure 10. Flow rate profile at the outlet in the center of the artery. The flow is measured at the coordinate point shown in the left frame. The right frame shows how the flow rate is modified due to the stent in the artery.

Since the main purpose of introducing the stent is to reduce the flow in the aneurysm, the results indicate that the stronger reduction is obtained using a completely extended Endeavor stent into the artery. The details of the fluid velocity in the aneurysm are displayed in Figure 11 for all cases of study.

The velocity field within the artery at different times (from 1.0 to $2.5 \mathrm{~s}$ as in Figure 8) are shown in Figures 12-16 for cases 1, 2, 3, 4, 5, respectively. In all runs, the highest velocity always occurs near the center of the artery in accordance with the theory of fluid flow in a channel or pipe. When the stent is present, the highest velocity is achieved in the stent zone due to the reduction of the transversal area. The greater increase of the fluid velocity in the stent zone for case 3 is clearly shown in Figure 14. The average velocity of the fluid in the artery is about $35 \mathrm{~cm} / \mathrm{s}$. However, in the case where the Endeavor stent is not completely extended the fluid velocity at the center of the stent reaches a velocity as high as $1 \mathrm{~m} / \mathrm{s}$. In all cases, the stent is seen to canalize the blood flow, avoiding that it passes through the aneurysm. This is shown in Figures 14-16, where the velocity into the aneurysm is clearly reduced compared to the velocity in the artery. Compared to the cases with stents, the velocity is always greater and even it is seen to increase with time in the aneurysm when the artery is free of stent, as shown in Figure 12.

Figure 17 depicts the velocity vectors in the aneurysm for the case when the artery is free of stent. The formation of a vortical flow is evident, which then increases with time in each cardiac cycle as shown by the size of the vectors in the middle and right frames. Each frame corresponds to one cardiac cycle according to Figure 8.

In contrast, when a stent is introduced in the artery, the vortical flow shown in Figure 17 disappears as it is evident from Figures 18-21 corresponding to cases 2 to 5 , respectively.

\subsection{Pressure}

The pressure at the inlet of the artery just before the stent zone provides a measure 

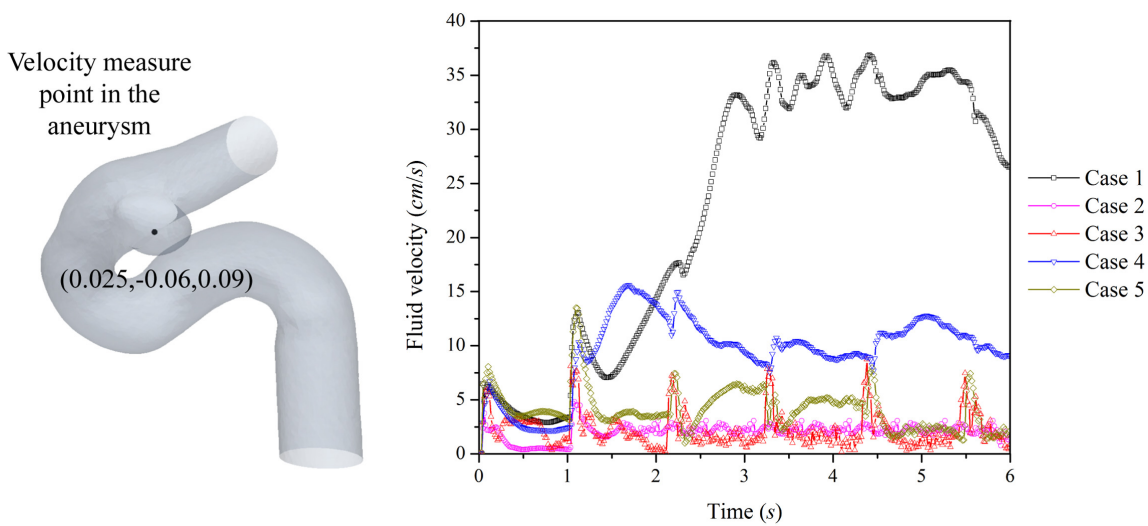

Figure 11. Flow rate profiles in the aneurysm as measured at the coordinate point depicted in the left frame. The right frame shows how the flow rate is modified due to the presence of the stent.

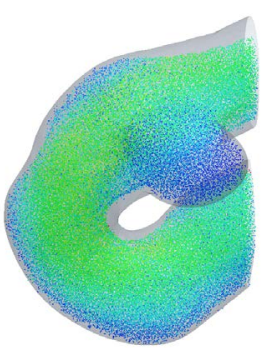

$t=1.0 \mathrm{~s}$

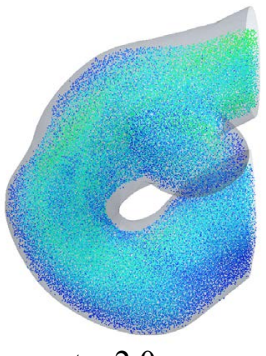

$t=2.0 \mathrm{~s}$

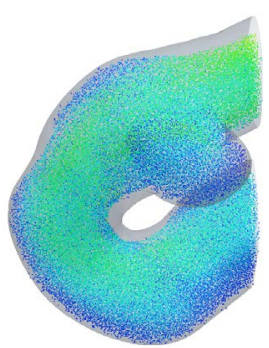

$t=1.5 \mathrm{~s}$

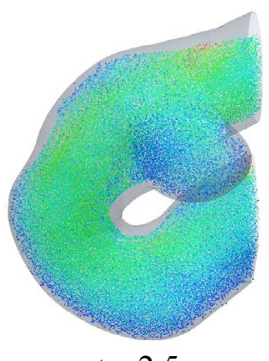

$t=2.5 \mathrm{~s}$

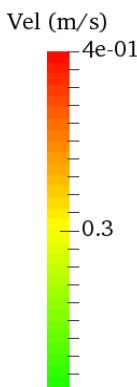

$-0.2$
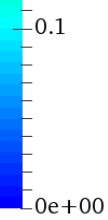

Figure 12. Velocity field in the artery without stent for one cardiac cycle from 1.0 to $2.5 \mathrm{~s}$, for the inlet flow rate shown in Figure 8.

of how the presence of the stent affects the artery internal pressure. In all cases, the fluid internal pressure increases when the stent is inserted. If it is desired that the internal pressure keeps almost constant before and after the stent zone, then the Endeavor stent is the best option provided that it is completely extended. However, if it is not completely extended this stent represents the worst option because it produces the highest increase in pressure compared with all other cases. The pressure profiles at the inlet of the artery are shown in Figure 22.

The pressure at the outlet of the artery provides information on the loss of pressure due to the stent configuration. For case 1, where the artery is free of stent no loss of pressure is seen because the flow is not hindered by an obstacle. 

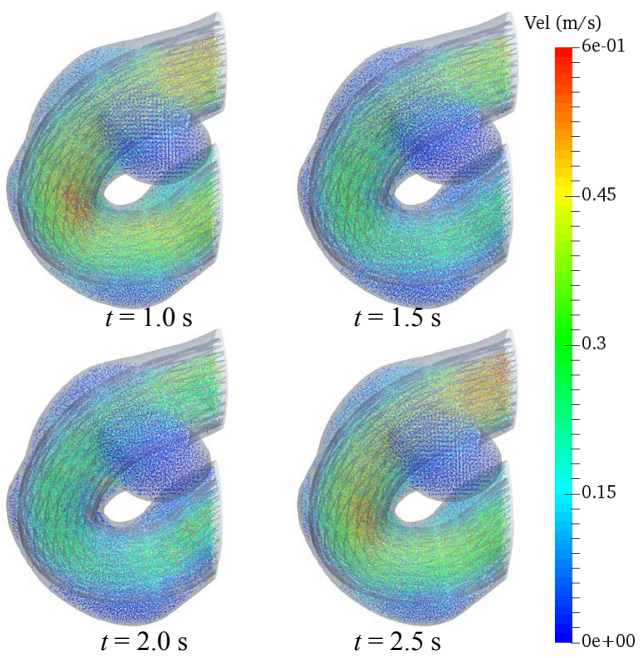

Figure 13. Velocity field into the artery with an Endeavor stent completely extended.
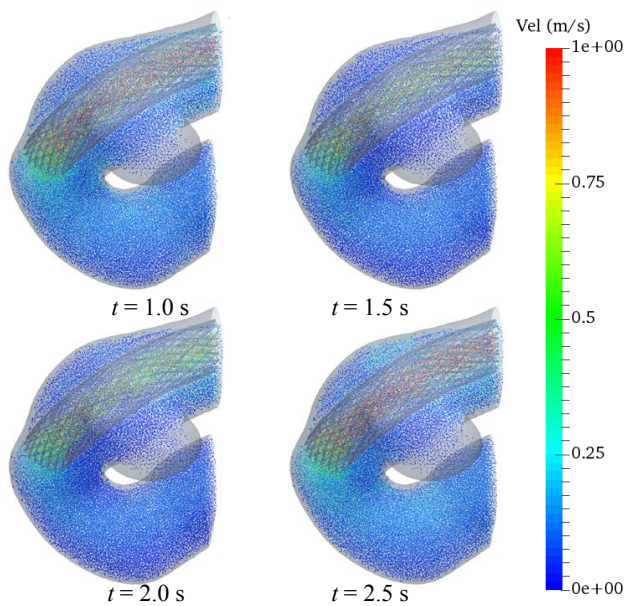

Figure 14. Velocity field into the artery with an Endeavor stent not completely extended.
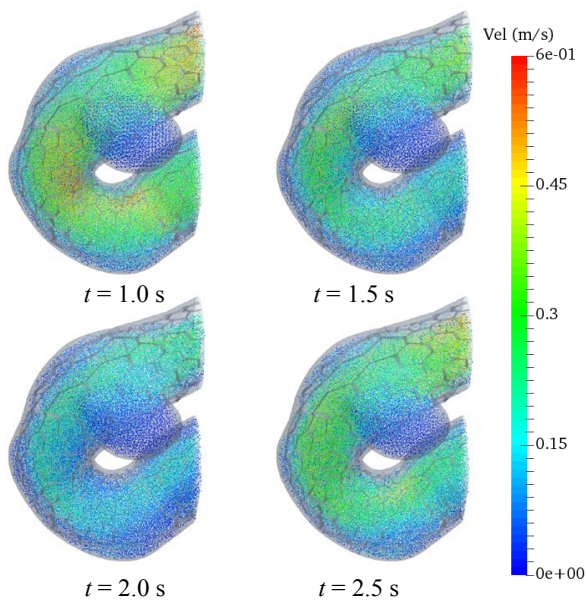

Figure 15. Velocity field into the artery with an octogonal stent completely extended. 

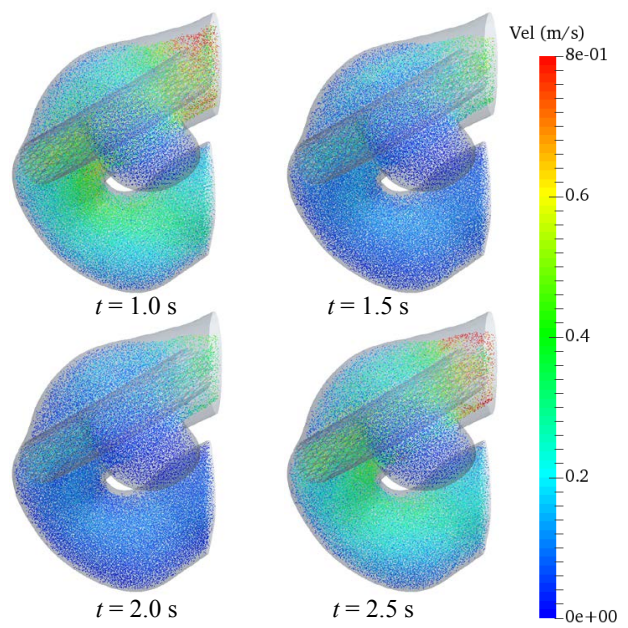

Figure 16. Velocity fields into the artery with an octogonal stent not completely extended.

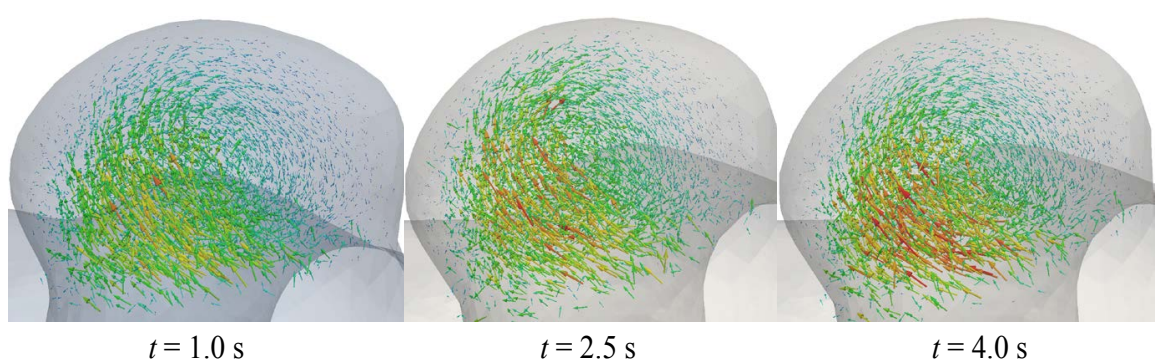

Figure 17. Vorticity field in the aneurysm when the artery is free of stent. The internal velocity increases with time as shown by the size of the velocity vectors.

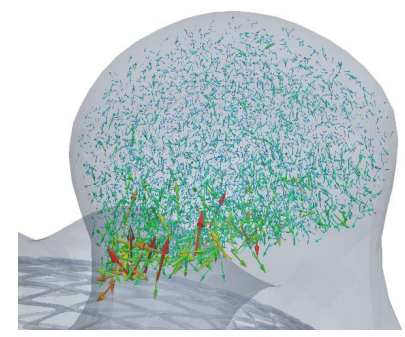

Figure 18. Velocity field in the aneurysm for case 2 at $t=2.5 \mathrm{~s}$.

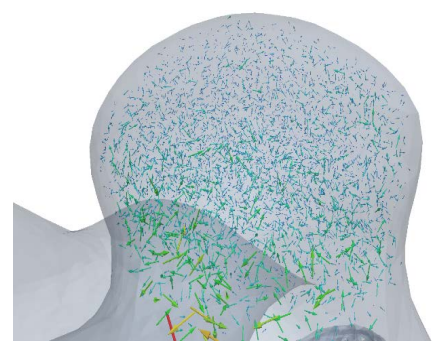

Figure 19. Velocity field in the aneurysm for case 3 at $t=2.5 \mathrm{~s}$.

In case 2 with the Endeavor stent completely extended, the pressure loss is about $300 \mathrm{~Pa}$ due to the presence of the stent, while in case 3 with the Endeavor stent 


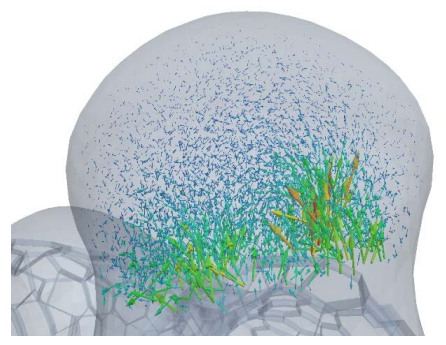

Figure 20. Velocity field in the aneurysm for case 4 at $t=2.5 \mathrm{~s}$.

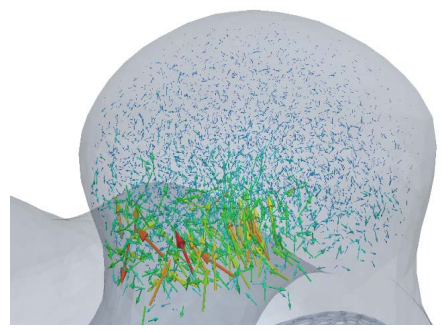

Figure 21. Velocity field in the aneurysm for case 5 at $t=2.5 \mathrm{~s}$.
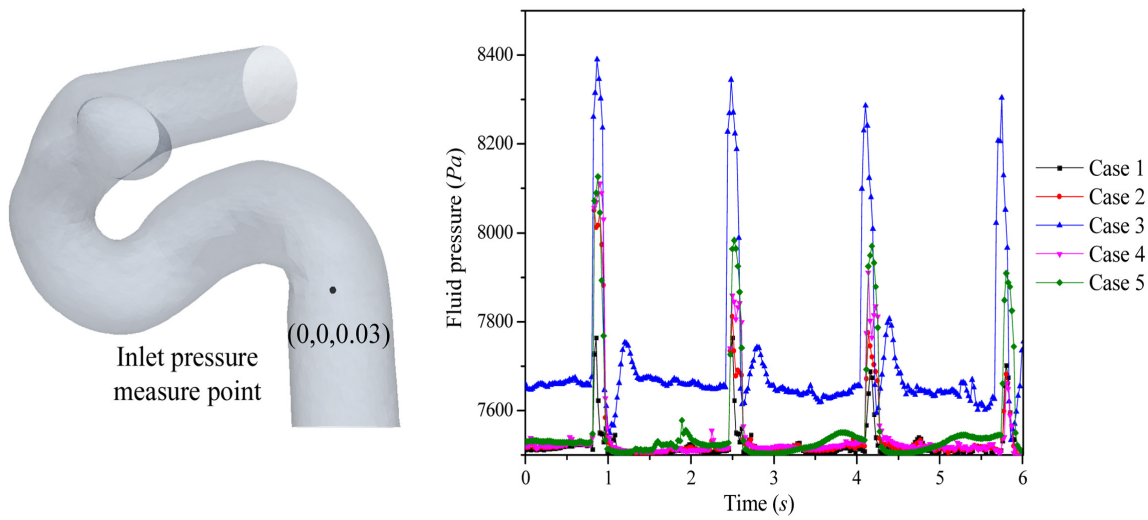

Figure 22. Internal artery fluid pressure just before the stent zone. In all cases, an increase of the pressure occurs when the stent is introduced in the artery.

not completely extended, the pressure loss increases to about 489 Pa. For comparison, cases 4 and 5 have a pressure loss of about 523 and $572 \mathrm{~Pa}$, respectively. The details of the fluid pressure at the outlet as a function of time are shown in Figure 23.

The pressure in the aneurysm has a similar behavior to the pressure at the outlet even in the cases where the stent is present within the artery, which is in good accordance with Pascal's law [25] [26]. The fluid pressure profile into the aneurysm is shown in Figure 24. The resulting pressure field through the artery at three different times into a cardiac cycle is shown in Figures 25-29. The pressure fields shown in these figures are in good agreement with the results described in Section 3.1.

\subsection{Wall Shear Stress Tensor}

For the analysis of the wall shear stress tensor, we follow Tanemura et al. [27] for 


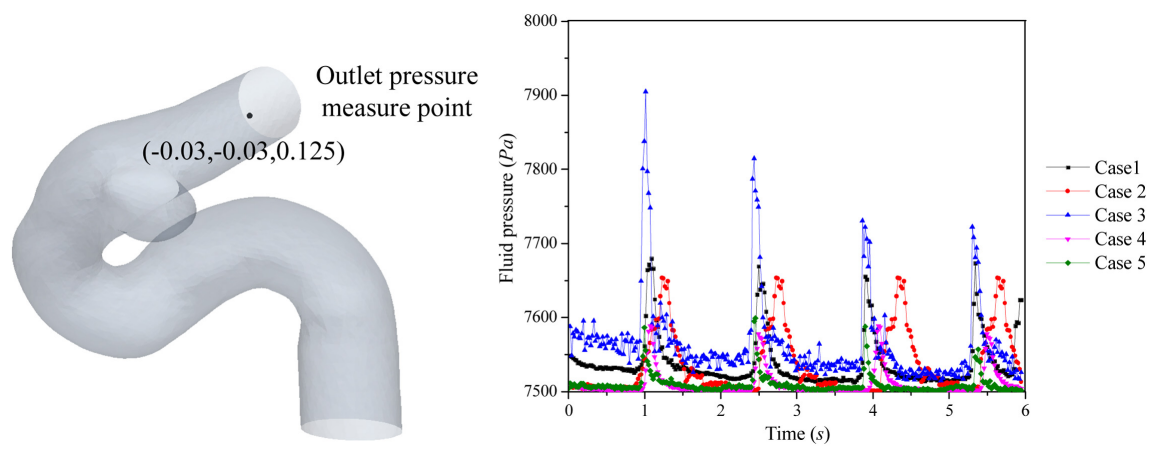

Figure 23. Internal artery fluid pressure at the outlet. In all cases, a pressure loss occurs in the presence of a stent, while no loss is observed without a stent.
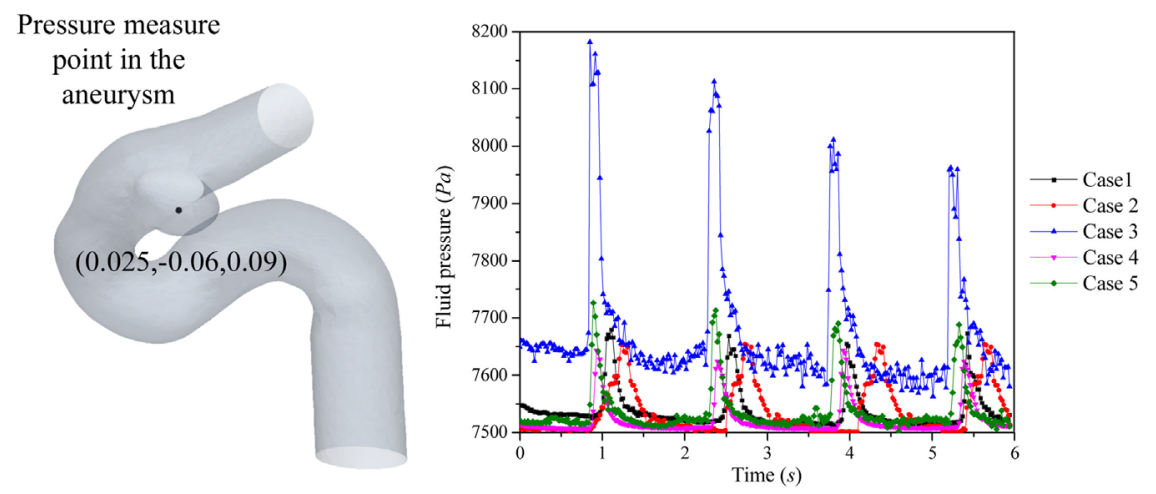

Figure 24. Arterial fluid pressure in the aneurysm. The pressure in the aneurysm has a similar behavior to the outlet pressure.
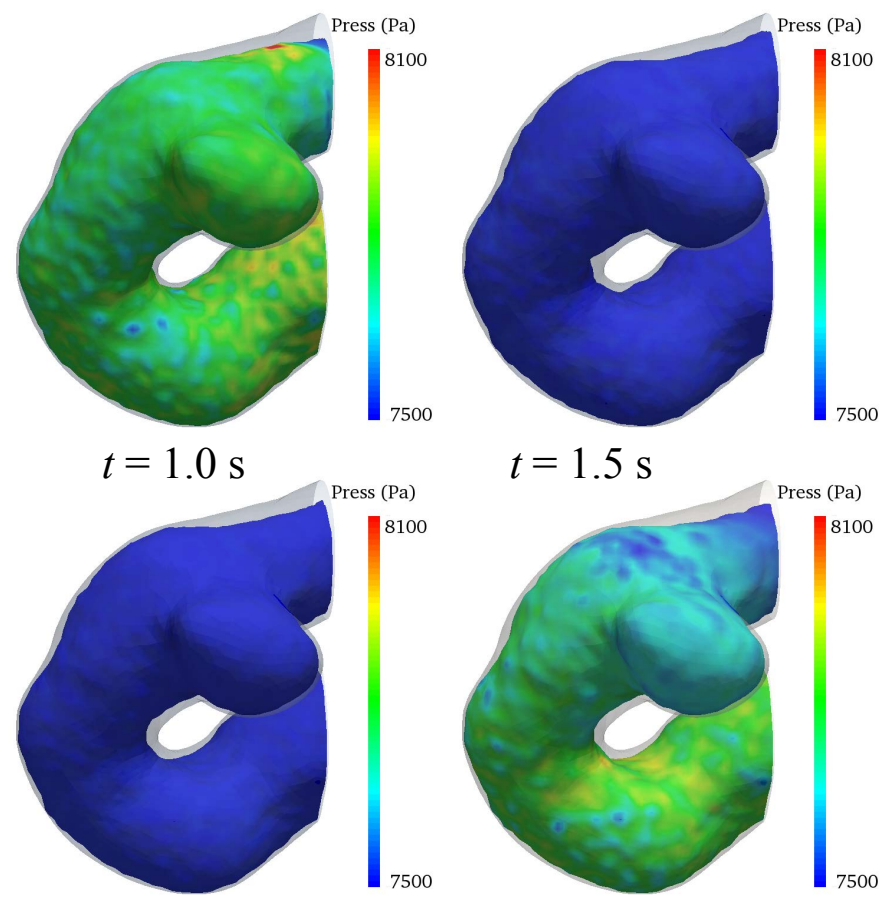

$$
t=2.0 \mathrm{~s}
$$

$$
t=2.5 \mathrm{~s}
$$

Figure 25. Pressure field in the artery for case 1. 

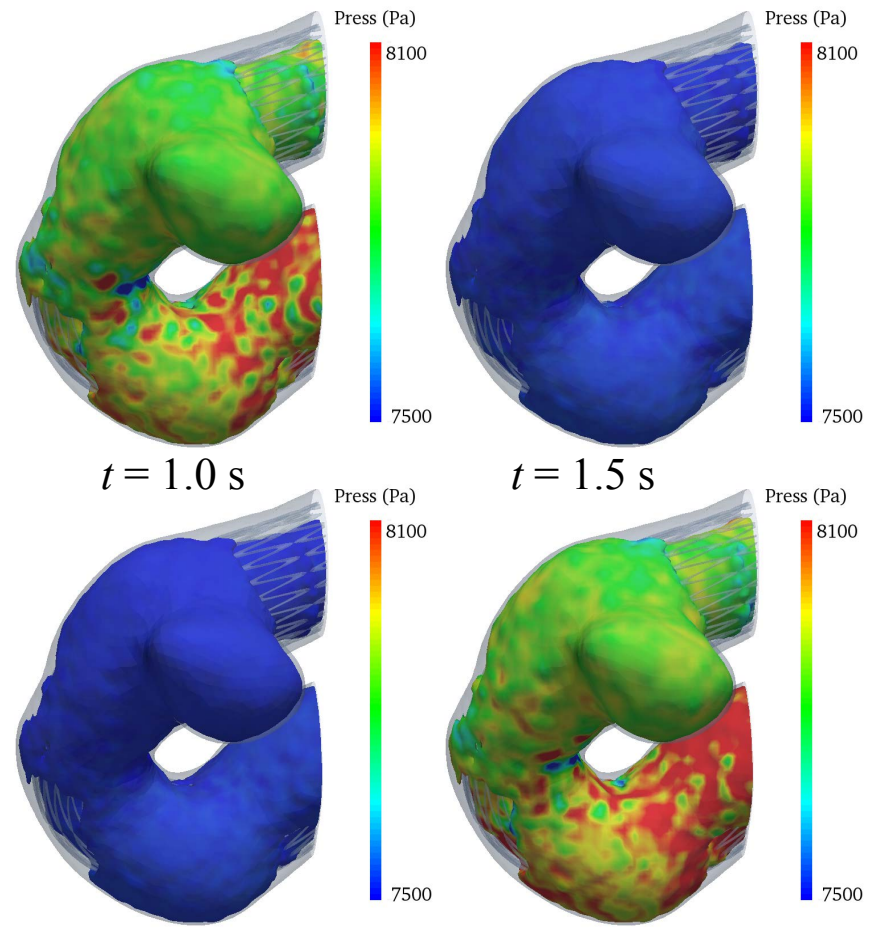

$t=2.0 \mathrm{~s}$

$t=2.5 \mathrm{~s}$

Figure 26. Pressure field in the artery for case 2.
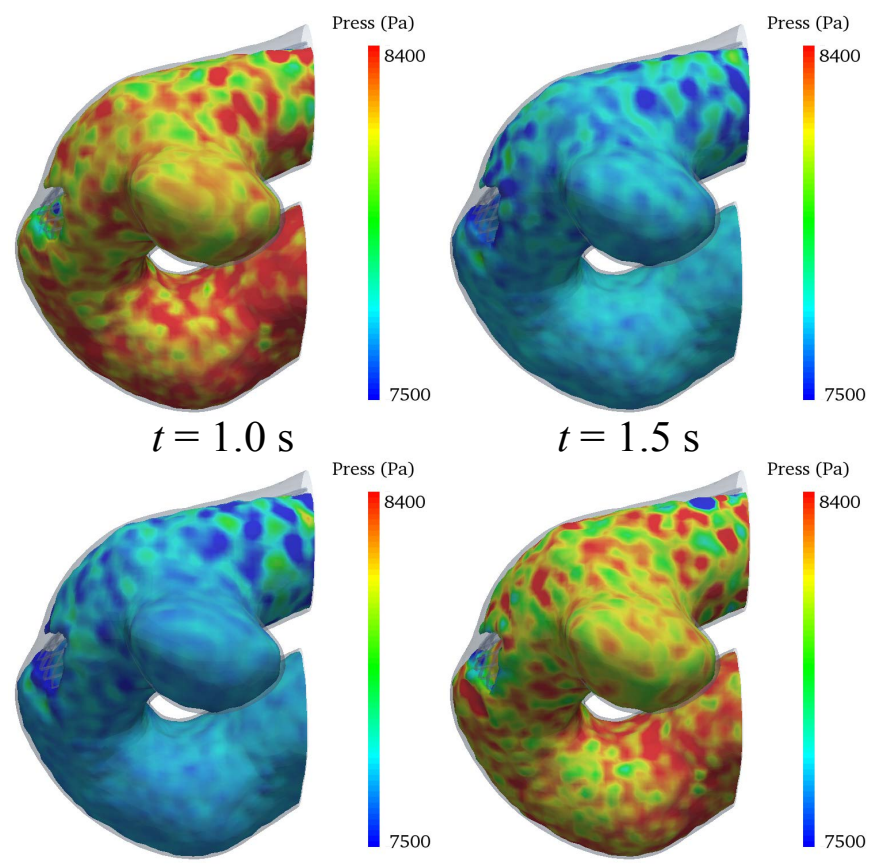

$t=2.0 \mathrm{~s}$

$$
t=2.5 \mathrm{~s}
$$

Figure 27. Pressure field in the artery for case 3.

the calculation of the hemodynamic parameters from the simulated flow fields, namely the wall shear stress (WSS), the oscillatory shear index (OSI), the WSS 

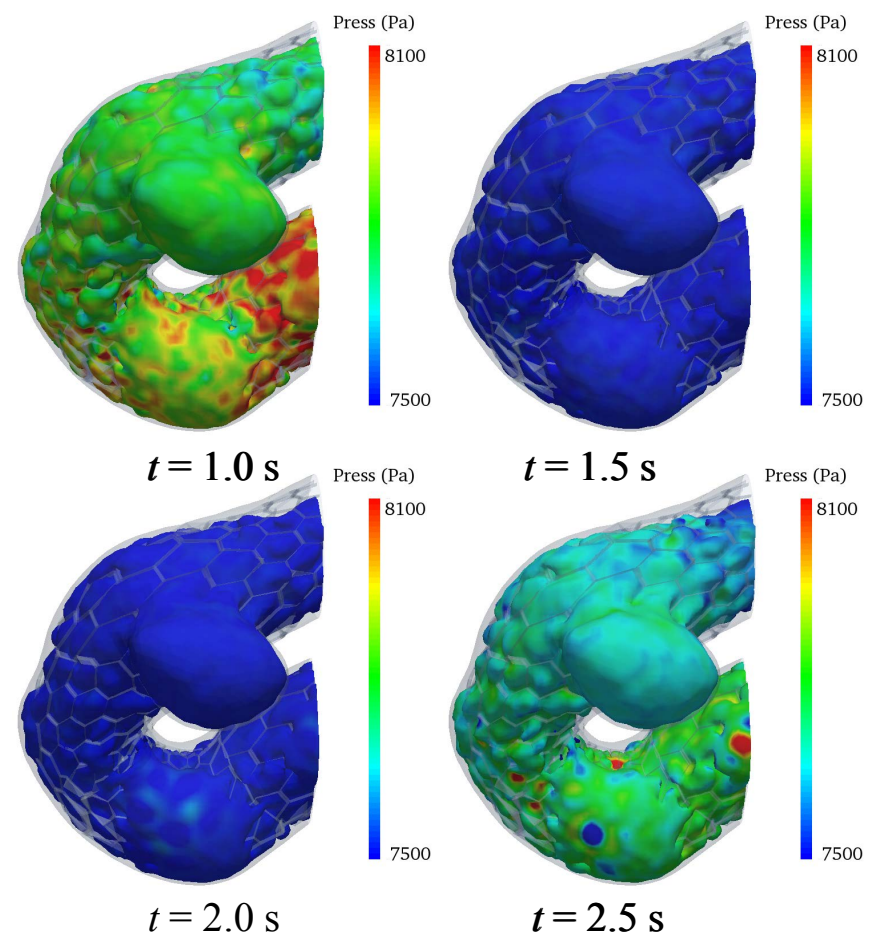

Figure 28. Pressure field in the artery for case 4.
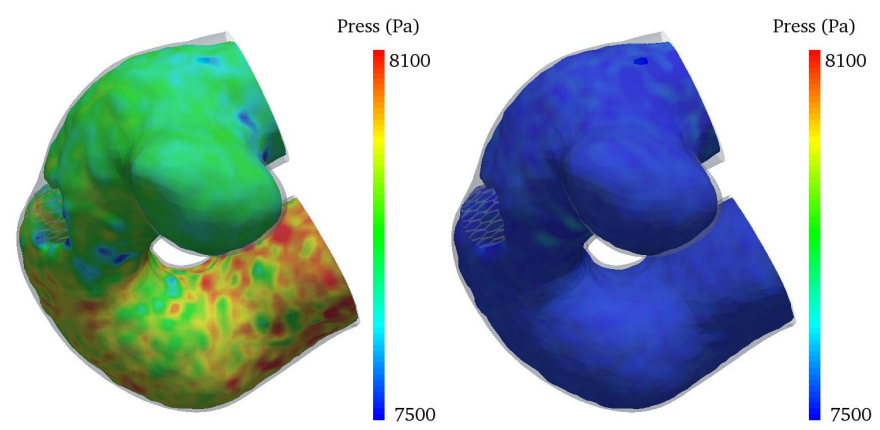

$$
t=1.0 \mathrm{~s}
$$

$$
t=1.5 \mathrm{~s}
$$

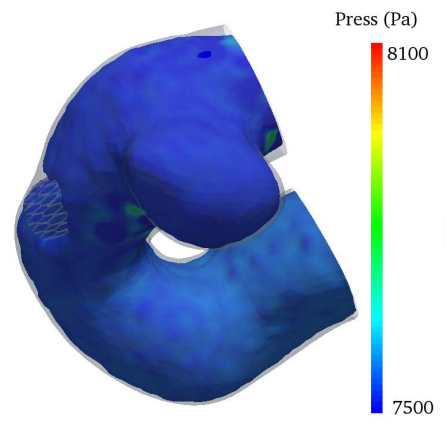

$$
t=2.0 \mathrm{~s}
$$

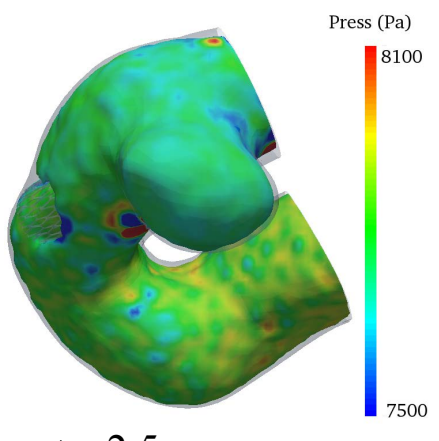

$$
t=2.5 \mathrm{~s}
$$

Figure 29. Pressure fields in the artery for case 5.

gradient (WSSG), the relative residence time (RRT) and the flow velocity (FV). To evaluate the hemodynamic effect of the stent placement, these hemodynamic parameters were compared for identical models with and without stent. For the 
details of the calculation of the hemodynamic parameters we refer to Tanemura et al. [27]. The results listed in Table 2 were calculated from the numerical results in the aneurysm region. However, the WSS in the artery is shown in Figure 30 at $t=2.5 \mathrm{~s}$. In all cases, the WSS is reduced when the stent is placed within the artery compared to the case when the artery is free of stent. The WSS value of 7.24 Pa predicted by our calculations for Case 1 with no stent compares qualitatively well with those reported by recent CFD simulations of blood flow in cerebral aneurysms, which range between 10.09 and $5.23 \mathrm{~Pa}$ [28].

In order to analyze the flow of blood around the stent, a singular section was chosen at the inlet of the aneurysm. The singular section is shown in Figure 31 for a completely extended octagonal stent. The numerical results clearly show that a turbulent flow is formed when the blood is blocked by the stent in the streamwise direction. However, this regime becomes laminar just before to be blocked again by the next wall of the stent as is shown in Figure 32. This special case was run with 2 million particles and the same parameters listed in the Table 1 .

Table 2. Hemodynamics parameters for each case of study.

\begin{tabular}{cccccc}
\hline Parameter & Case 1 & Case 2 & Case 3 & Case 4 & Case 5 \\
\hline WSS $(\mathrm{Pa})$ & 7.24 & 3.83 & 11.1 & 2.60 & 0.866 \\
OSI & 0.16 & 0.208 & 0.269 & 0.066 & 0.187 \\
$\begin{array}{c}\text { WSSG } \\
(\mathrm{Pa} / \mathrm{mm})\end{array}$ & 12.92 & 7.09 & 19.4 & 3.95 & 1.37 \\
$\mathrm{RRT}\left(\mathrm{m}^{2} / \mathrm{N}\right)$ & 0.306 & 0.671 & 2.93 & 0.666 & 2.77 \\
$\mathrm{FV}(\mathrm{m} / \mathrm{s})$ & 0.589 & 0.100 & 0.100 & 0.263 & 0.082 \\
\hline
\end{tabular}

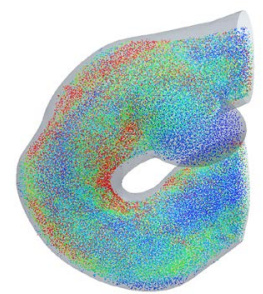

Case 1

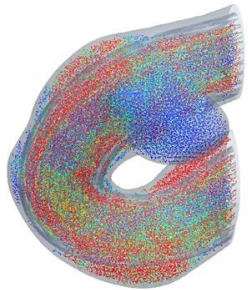

Case 2

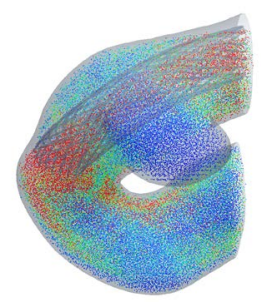

Case 3

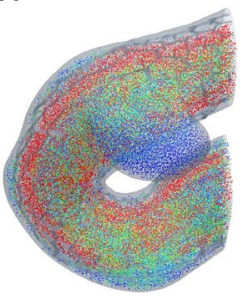

Case 4

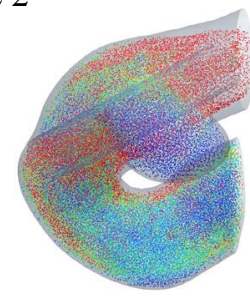

Case 5

Figure 30. The WSS through the artery. The WSS is reduced into the aneurysm in all cases with stent. However, the value of the WSS increases around the artery in the stent zone. 


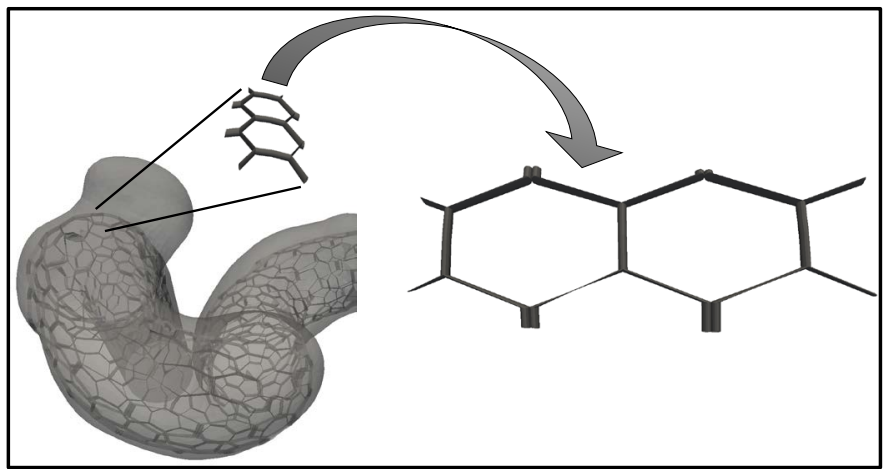

Figure 31 . Isolated singular section employed to analyze the fluid flow through the mesh of the stent.

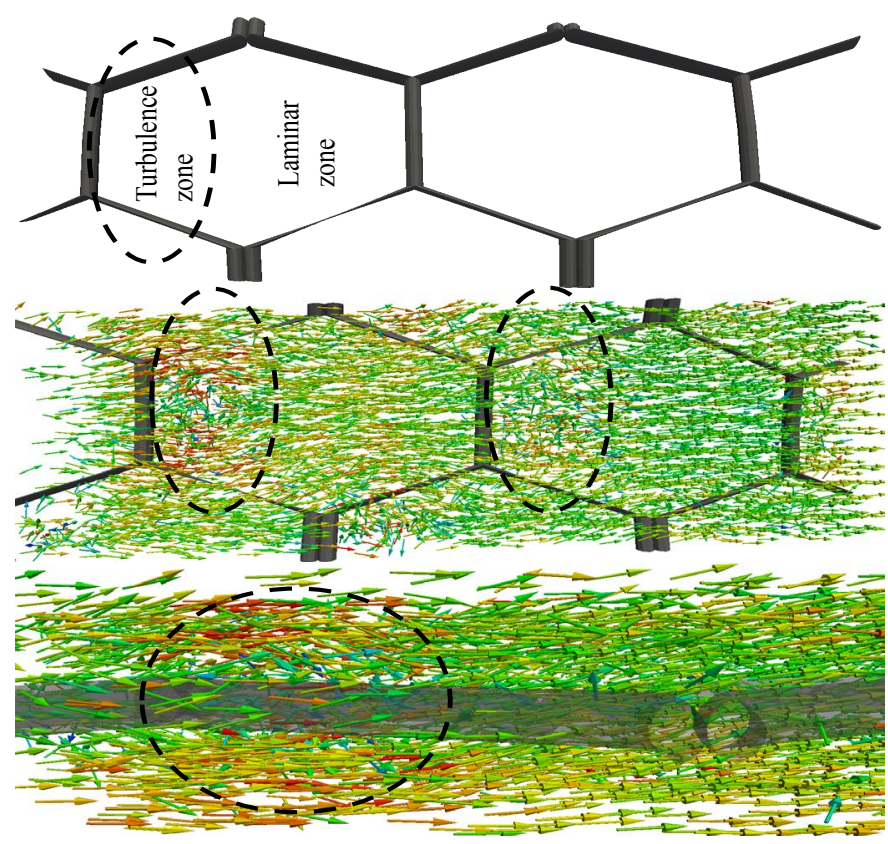

Figure 32. Velocity vectors in the isolated singular section shown in Figure 31.

\section{Concluding Remarks}

We have presented exploratory SPH calculations of blood flow through a brain vascular aneurysm using Smoothed Particle Hydrodynamics (SPH) methods. The initial conditions were constructed from angiographic images of a real patient with an aneurysm. The flow structure within the aneurysm was investigated in terms of the flow velocity, the internal pressure and the wall shear stresses for five model cases of flow with and without stent. The first case corresponds to flow without stent, while the other four cases correspond to flow in the presence of an Endeavor and an octagonal stent, when they are completely extended and partially extended within the artery.

The results indicate that the flow downstream the aneurysm is highly modified by the stent configuration. The thickness of the stent canalizes the flow within the artery due to a reduction of the transversal area, resulting in an in- 
creasing flow velocity and a pressure loss. Moreover, when a stent is introduced in the artery, significant changes of the velocity occur in the aneurysm. When the Endeavor stent is completely extended within the artery, the flow in the aneurysm is seen to decrease by more than $90 \%$ compared to about $70 \%$ for the case of an Endeavor stent not completely extended. Similar drops of the flow velocity were also observed for an octagonal stent. More importantly, the run with no stent predicted the development of turbulent flow within the aneurysm, which was also seen to increase with time. Conversely, this vortical flow was seen to strongly decrease and even disappear when a stent is inserted into the artery. On the other hand, the pressure losses across the aneurysm were seen to be slightly higher for the cases with an octagonal stent. Considering that the main purpose of introducing a stent is that of reducing the flow in the aneurysm, the results indicate that the best choice is to use an Endeavor stent completely extended into the artery. Moreover, if the internal pressure has to remain almost constant before and after the stent, the best option is again the Endeavor stent completely extended. However, if this stent is not completely extended then it becomes the worst option since it produces the highest increase in pressure compared to all other cases.

Future studies in this direction will include models of brain vascular flow with different parameters and different patient aneurysm images. Further testing of the code will also include direct comparison with true medical data.

\section{Acknowledgements}

We acknowledge the Universidad Autónoma Metropolitana-Azcapotzalco (UAMA) for partial support.

\section{Conflicts of Interest}

The authors declare no conflicts of interest regarding the publication of this paper.

\section{References}

[1] de Rooij, N.K., Linn, F.H.H., van der Plas, J.A., Algra, A. and Rinkel, G.J.E. (2007) Incidence of Subarachnoid Haemorrhage: A Systematic Review with Emphasis on Region, Age, Gender and Time Trends. Journal of Neurology and Neurosurgery Psychiatry, 78, 1365-1372. https://doi.org/10.1136/jnnp.2007.117655

[2] Wiebers, D.O. (2003) Unruptured Intracranial Aneurysms: Natural History, Clinical Outcome, and Risks of Surgical and Endovascular Treatment. The Lancet, 362, 103-110. https://doi.org/10.1016/S0140-6736(03)13860-3

[3] Dhar, S., Tremmel, M., Mocco, J., Kim, M., Yamamoto, J., Siddiqui, A.H., et al. (2008) Morphology Parameters for Intracranial Aneurysm Rupture Risk Assessment. Neurosurgery, 63, 185-197. https://doi.org/10.1227/01.NEU.0000316847.64140.81

[4] Mocco, J., Brown, R.D., Torner, J.C., Capuano, A.W., Fargen, K.M., Raghavan, M.L., et al. (2018) Aneurysm Morphology and Prediction of Rupture: An International Study of Unruptured Intracranial Aneurysms Analysis. Neurosurgery, 82, 
491-496. https://doi.org/10.1093/neuros/nyx226

[5] Almefty, K. and Spetzler, R.F. (2014) Management of Giant Internal Carotid Artery Aneurysms. World Neurosurgery, 82, 40-42. https://doi.org/10.1016/j.wneu.2013.03.064

[6] Hanel, R.A. and Spetzler, R.F. (2008) Surgical Treatment of Complex Intracranial Aneurysms. Neurosurgery, 62, 1289-1299. https://doi.org/10.1227/01.neu.0000333794.13844.d9

[7] Kalani, Y.S.M., Zabramski, J.M., Hu, Y.C. and Spetzler, R.F. (2013) Extracranial-Intracranial Bypass and Vessel Occlusion for the Treatment of Unclippable Giant Middle Cerebral Artery Aneurysms. Neurosurgery, 72, 428-435. https://doi.org/10.1227/NEU.0b013e3182804381

[8] Chalouhi, N., Jabbour, P., Tjoumakaris, S., Starke, R.M., Dumont, A.S., Liu, H., et al. (2013) Treatment of Large and Giant Intracranial Aneurysms: Cost Comparison of Flow Diversion and Traditional Embolization Strategies. World Neurosurgery, 82, 1-6.

[9] Taussky, P., Tawk, R.G., Miller, D.A., Freeman, W.D. and Hanel, R.A. (2013) New Therapies for Unruptured Intracranial Aneurysms. Neurologic Clinics, 31, 737-747. https://doi.org/10.1016/j.ncl.2013.03.011

[10] Auricchio, F., Conti, M., Morganti, S. and Reali, A. (2014) Simulation of Transcatheter Aortic Valve Implantation: A Patient-Specific Finite Element Approach. Computer Methods in Biomechanicsaand Biomedical Engineering, 17, 1347-1357. https://doi.org/10.1080/10255842.2012.746676

[11] Mine, B., Bonnet, T., Vazquez-Suarez, J.C., Iosif, C. and Lubicz, B. (2018) Comparison of Stents Used for Endovascular Treatment of Intracranial Aneurysms. Expert Review of Medical Devices, 15, 793-805. https://doi.org/10.1080/17434440.2018.1538779

[12] Song, Y., Choe, J., Liu, H., Park, K.J., Yu, H., Lim, O.K., et al. (2016) Virtual Stenting of Intracranial Aneurysms: Application of Hemodynamic Modification Analysis. Acta Radiologica, 57, 992-997. https://doi.org/10.1177/0284185115613653

[13] Takizawa, K., Schjodt, K., Puntel, A., Kostov, N. and Tezduyar, T.E. (2012) Patient-Specific Computer Modeling of Blood Flow in Cerebral Arteries with Aneurysm and Stent. Computional Mechanics, 50, 675-686. https://doi.org/10.1007/s00466-012-0760-4

[14] Monaghan, J.J. (1992) Smoothed Particle Hydrodynamics. Annual Review of Astronomy and Astrophysics, 30, 543-574. https://doi.org/10.1146/annurev.aa.30.090192.002551

[15] Müller, M., Schirm, S. and Teschner, M. (2018) Interactive Blood Simulation for Virtual Surgery Based on Smoothed Particle Hydrodynamics. Technology and Health Care, 12, 25-31.

[16] Caballero, A., Mao, W., Liang, L., Oshinski, J., Primiano, C., McKay, R., et al. (2017) Modeling Left Ventricular Blood Flow Using Smoothed Particle Hydrodynamics. Cardiovascular Engineering and Technology, 8, 465-479. https://doi.org/10.1007/s13239-017-0324-Z

[17] Babiker, M.H., Chong, B., Gonzalez, L.F., Cheema, S. and Frakes, D.H. (2013) Finite Element Modeling of Embolic Coil Deployment: Multifactor Characterization of Treatment Effects on Cerebral Aneurysm Hemodynamics. Journal of Biomechanics, 46, 2809-2816. https://doi.org/10.1016/j.jbiomech.2013.08.021

[18] Ishizawa, K., Komori, T., Shimada, T., Arai, E., Imanaka, K., Kyo, S., et al. (2005) Hemodynamic Infarction of the Spinal Cord: Involvement of the Gray Matter plus 
the Border-Zone between the Central and Peripheral Arteries. Spinal Cord, 43, 306-310. https://doi.org/10.1038/sj.sc.3101671

[19] Li, Z. and Kleinstreuer, C. (2005) Fluid-Structure Interaction Effects on Sac-Blood Pressure and Wall Stress in a Stented Aneurysm. Journal of Biomechanical Engineering, 127, 662-671. https://doi.org/10.1115/1.1934040

[20] Long, Q., Xu, X.Y., Collins, M.W., Griffith, T.M. and Bourne, M. (1998) The Combination of Magnetic Resonance Angiography and Computational Fluid Dynamics: A Critical Review. Critical Reviews in Biomedical Engineering, 26, 227-274. https://doi.org/10.1615/CritRevBiomedEng.v26.i4.10

[21] Qin, Y., Wu, J., Hu, Q., Ghista, D.N. and Wong, K.K.L. (2017) Computational Evaluation of Smoothed Particle Hydrodynamics for Implementing Blood Flow Modelling through CT Reconstructed Arteries. Journal of X-Ray Science and Technology, 25, 213-232. https://doi.org/10.3233/XST-17255

[22] Liu, G.R. and Liu, M.B. (2003) Smoothed Particle Hydrodynamics: A Meshfree Particle Method. World Scientific, Singapore. https://doi.org/10.1142/5340

[23] Crespo, A.J.C., Gómez-Gesteira, M. and Dalrymple, R.A. (2007) Boundary Conditions Generated by Dynamic Particles in SPH Methods. Computers, Materials \& Continua, 5, 173-184.

[24] Alvarado-Rodríguez, C.E., Klapp J., Sigalotti, L.Di G., Domínguez, J.M. and de la Cruz Sánchez, E. (2017) Nonreflecting Outlet Boundary Conditions for Incompressible Flows Using SPH. Computers \& Fluids, 159, 177-188. https://doi.org/10.1016/j.compfluid.2017.09.020

[25] Gotoh, H., Shibahara, T. and Sakai, T. (2001) Sub-Particle-Scale Turbulence Model for the MPS Method-Lagrangian Flow Model for Hydraulic Engineering. Computational Fluid Dynamics Journal, 9, 339-347.

[26] Lo, E.Y.M. and Shao, S. (2002) Simulation of Near-Shore Solitary Wave Mechanics by an Incompressible SPH Method. Applied Ocean Research, 24, 275-286.

[27] Tanemura, H., Ishida, F., Miura, Y., Umeda, Y., Fukazawa, K., Susuki, H., Sakaida, H., Matsushima, S., Shimosaka, S. and Taky, W. (2013) Change in Hemodynamics after Placing Intercranial Stents. Neurologia Medico-Chirurgica, 53, 171-178.

[28] Shishir, S.S., Miah, Md. A.K., Islam, A.K.M.S. and Hasan, A.B.M.T. (2015) Blood Flow Dynamics in Cerebral Aneurysm-A CFD Simulation. Procedia Engineering, 105, 919-927. https://doi.org/10.1016/j.proeng.2015.05.116 